\title{
Comparación en las estructuras de colaboración y pautas de citación entre áreas científicas a través del ARS
}

\author{
Mayte López Ferrer - Instituto de Gestión de la Innovación y del Conocimiento \\ (INGENIO) CSIC-UPV ${ }^{1}$
}

\section{Resumen}

Esta investigación se enmarca en los estudios sociométricos de literatura científica. En concreto en la aplicación del análisis de redes a la colaboración científica y las pautas de citación entre investigadores para comparar tres áreas temáticas: psiquiatría, psicología general y psicología experimental. Los objetivos son aplicar el análisis de redes para acotar universos de conocimiento independientes pero lo suficientemente próximos y relacionados como para estudiarlos en paralelo, buscar sus similitudes y diferencias en base a los indicadores bibliométricos y de red obtenidos. Se concluye que el análisis de redes aplicado a los sistemas de clasificación de las bases de datos es útil para acotar universos de conocimiento que trascienden los propios sistemas de clasificación siempre más simplistas; que los gráficos de redes permiten objetivar grupos de trabajo y dinámicas en ellos que de otro modo sería difícil descubrir, para ello resulta además especialmente útil superponer las redes de colaboración con las de citas puesto que las primeras representan relaciones explícitas y las segundas relaciones implícitas, pero ambas sirven para posicionar autores y grupos entre sí. Finalmente, se apunta la necesidad de una flexibilización en los indicadores de seguimiento y evaluación de las diferentes áreas científicas, que tenga en cuenta las características propias de las diversas disciplinas, también la idoneidad de la obtención de nuevos indicadores basados no tanto en la producción de autores estudiados unitariamente como su capacidad de intermediación con otros actores, es decir, de articulación del sistema de investigación.

Palabras clave: Redes temáticas - Coautoría - Estructuras de colaboración Pautas de citación - Psiquiatría - Psicología

\begin{abstract}
This research is framed in sociometric studies above bibliography. In particular, in the application of social Networks to co authorship, and the patterns of citation between researchers, focused in comparison of Psychiatry and Psychology and areas. The objectives are: the application of social network analysis to retrieval information in a multidisciplinary database, due to subject headings lists are considered to be insufficient and inflexible to describe relationship between sciences; and the establishment of similarities and differences between the three areas according to bibliometric and network indicators. The Social Network analysis used in this work to select scientific articles of a discipline avoids the rigidity of retrieval information based in a preselected set of subjects that never explains the overlapping and that could to mutilate extensions of some of them or just simply to produce silence before incipient relations. Also, network graphs show working groups that otherwise would be difficult to discover. In addition, it is especially useful to superpose networks of co authorship with those of cited references patterns because the first represent explicit relations and the second one represent implicit relations, but both serve to compare each author or group to the others. And finally, the necessity of an adaptation of the monitoring and evaluation
\end{abstract}

\footnotetext{
${ }^{1}$ Enviar correspondencia a: (mlopezf@ingenio.upv.es)
} 
indicators of the different scientific areas is indicated, which considers the own characteristics of the diverse disciplines, and they must be based not as much in the productivity of authors studied unitarily as its capacity of intermediation with other actors and their capacity of articulation of the research system.

Key words: Subject networks - Co authorship - Collaborative structures Patterns of citation - Psychiatry - Psychology.

\section{I ntroducción}

El análisis de redes sociales ha supuesto una revolución en los estudios bibliométricos de la actividad científica. El término bibliometría se acuñó frente a bibliografía estadística para conceptualizar la aplicación de los métodos estadísticos y matemáticos a las publicaciones científicas como método para explicar el desarrollo de las disciplinas científicas, fundamentalmente mediante el recuento y análisis de los elementos de dichas comunicaciones (Pritchard, 1969). Posteriormente a este campo semántico se han añadido nuevos términos como cienciometría, informetría, cibermetría, etc. con las correspondientes precisiones, ampliaciones y restricciones de sus significados fundamentalmente para ampliar el objeto de estudio y considerar otros elementos de la comunicación científica susceptibles de explicar la actividad científica. En este trabajo nos referiremos a bibliometría o estudios bibliométricos por continuar siendo el más utilizado y conocido en la ciencia internacional (Jiménez, 2000).

El análisis de redes sociales, en adelante ARS, estudia de manera genérica las relaciones que se establecen entre elementos. Tanto los elementos como las relaciones admiten su aplicación a cualquier campo en el que se produzcan interacciones. Mientras que en los análisis sociales tradicionales, los elementos se estudian clasificándolos o agrupándolos en función de sus características (estratificación de una población en clases sociales, división en sexos, grupos de edad, localización geográfica, etc.) el ARS se fundamenta en la idea de que las estructuras de relaciones entre elementos explican mejor el conjunto, el entorno social y también a cada uno de los elementos, que los atributos de éstos tomados unitariamente.

Con el ARS los estudios bibliométricos han transformado completamente sus interrogantes, ya no preocupan tanto, por ejemplo, las producciones científicas de las instituciones sino sus redes de colaboración; o la calidad de las publicaciones basándose en los recuentos de las citas recibidas sino en los significados que estos los flujos de citación aportan para la comprensión de las interacciones entre 
disciplinas científicas. También las visualizaciones que proporciona el ARS han revalorizado los estudios bibliométricos dado su poder de comunicación de fenómenos que las matrices de datos no permiten intuir.

Las dos aplicaciones más generalizadas del ARS a la bibliometría son la elaboración de mapas de la ciencia, es decir, redes temáticas construidas normalmente a partir las citas (Boyack, 2005; Iñiguez, 2006; Leydesdorff, 2004; Leydesdorff, 2009; Moya-Anegón, 2004). Y los estudios de colaboración científica, fundamentada en redes personales para las que se toma como contenido de la relación la coautoría en publicaciones científicas (Acedo, 2006; Bozeman, 2004; Olmeda-Gomez, 2009; Perianes-Rodriguez, 2010). También se han analizado las redes personales a través de la copresencia en tribunales de tesis (Casanueva, 2007; Delgado, 2006; Olmeda-Gómez, 2009), ó en tribunales de oposición (Sierra, 2003) y quedan seguramente todavía muchas fuentes de información por explotar.

En su función evaluadora de la actividad científica los estudios bibliométricos siempre han tenido al investigador como unidad de análisis, considerado individualmente, puesto que los indicadores están diseñados para comparar investigadores entre sí. También cuando lo que se comparan son otras unidades definidas en el análisis: grupos, países, universidades, etc. se comparan estadísticos calculados a partir de los indicadores obtenidos de los investigadores individualmente pertenecientes a dichos grupos, países, o universidades. Con la situación actual, un proyecto de investigación competitiva que concurse para la obtención de financiación pública tendrá más garantías de éxito si está refrendado por un equipo cuyos individuos acumulen gran cantidad de méritos, lo que se conoce como excelencia del grupo de investigación, pero esa evaluación consiste en la adición de méritos individuales, sin que se hayan desarrollado herramientas que permitan valorar el grupo como equipo. De camino a ese posible nuevo sistema de evaluación de grupos es necesario describir para comprender las distintas formas de organización del trabajo y el sistema de reconocimientos en las distintas áreas de conocimiento.

El ARS, sin embargo, permite poner la atención no en los individuos sino en las estructuras en las que se insertan y su contexto. Cualquier conclusión obtenida desde esta perspectiva, quizás nuevos indicadores de actividad científica, tendrán una aplicación en políticas científicas a más largo plazo puesto, que actuarán sobre la estructura y no sobre los individuos. 
Algunos trabajos que confluyen desde la economía aplicada y la política científica han llevado a cabo ensayos para evaluar la eficiencia de los grupos de investigación. En ellos aplican criterios y técnicas de eficiencia en el uso de los recursos, tanto humanos como económicos y en los resultados. Entre los resultados se distinguen las publicaciones científicas y patentes como resultados científicotecnológicos, el personal formado y tesis doctorales como resultados formativos, y los contratos bilaterales con empresas como resultados socioeconómicos. Se observa que diferentes grupos de investigación presentan trayectorias particulares y se orientan hacia distintos tipos de resultados, por lo que se pone de manifiesto la necesidad de promover un análisis dinámico de la eficiencia ofrecida por cada grupo con el fin de diseñar políticas más adaptadas a las necesidades de los distintos tipos de grupos (Jiménez, 2007). También el ARS podría introducir en estos análisis de eficiencia factores a tener en cuenta. La cultura organizativa de los distintos grupos, su tamaño, estructura, composición, permeabilidad a nuevos miembros, etc. podría tener incidencia en su rendimiento, y, por tanto, abrir una posibilidad a los administradores de política científica para incentivar los comportamientos más eficientes como grupo.

El análisis de redes en política científica permite conjugar esa doble característica que Molina (2009) expone: una reducción débil de la realidad observada, y al mismo tiempo una operacionalización fuerte de las representaciones y sus propiedades. Una reducción débil porque las visualizaciones del fenómeno estudiado son fácilmente reconocibles por sus protagonistas y nos permiten incorporar a la representación muchas variables atributivas para matizar las observaciones, muchas de ellas obtenidas de la propia percepción del fenómeno por sus protagonistas. $\mathrm{Y}$, por otro lado, una operacionalización fuerte porque las propiedades matemáticas de los grafos aportan parámetros de medir, comparar e incluso experimentar con sus individuos y topologías.

El presente trabajo se enmarca en los estudios sociométricos de literatura científica. Se trata de la aplicación del ARS a tres aspectos de los estudios de literatura científica, el primero de ellos atañe a la delimitación de áreas científicas, el segundo a la coautoría y el tercero a las pautas de citación entre autores. Los objetivos del presente trabajo de investigación son:

1. Aplicar el ARS para acotar universos de conocimiento independientes pero lo suficientemente próximos y relacionados como para estudiarlos en paralelo, buscar sus similitudes y diferencias. 
2. Utilizar complementariamente las técnicas bibliométricas con el ARS en un estudio sobre coautoría y pautas de citación entre autores.

3. Establecer similitudes y diferencias en base a los indicadores obtenidos a partir del ARS entre las tres áreas temáticas propuestas a partir del objetivo inicial: Psiquiatría y Neurociencias, Psicología General y Psicología Experimental.

En el caso concreto de esta investigación se pretende presentar de forma panorámica los grupos de investigación de tres disciplinas, su distribución, su prominencia, sus interacciones o la ausencia de estas. Las poblaciones a estudiar son completamente desconocidas a priori y, por tanto, la selección es altamente objetiva puesto que se recupera de bases de datos de acceso público y con estrategias repetibles. Al utilizar fuentes documentales ya existentes y creadas para otros fines se supera una de las limitaciones que se reconoce a los estudios de ARS basados en datos obtenidos por medio de la observación del investigador: el hecho de que la recogida de datos siempre es parcial, porque la observación directa es compleja.

El obstáculo que supone la capacidad de los programas informáticos de análisis de redes sociales, que llegan a trabajar con redes de unos pocos miles de vértices como máximo, queda superada aquí al combinar la utilización de las variables relacionales con la variable atributiva de la producción científica, lo que justifica la eliminación de algunos actores, los de más baja producción, para permitir un estudio manejable (Lotka, 1926).

Destacamos dos limitaciones a tener en cuenta, recelo, por otro lado, no muy frecuente en los trabajos que aplican el ARS a la bibliometría. De una parte la idoneidad de la utilización de la coautoría como indicador de colaboración científica. Katz (1997) afirma que, aunque la colaboración se mide convencionalmente a través de las coautorías, es solamente un indicador parcial que se debe tratar con precaución puesto que lo que es o no colaboración entre científicos es en gran parte una convención social que varía entre instituciones, disciplinas, países y cambia además como resultado de las políticas de las agencias de la financiación y la estimulación de los gobiernos a la colaboración.

De otra parte, la indefinición del significado de una cita entre autores o su ausencia. Según Vinkler (1987) las motivaciones para citar un trabajo anterior son de dos tipos: profesionales y sociales. Las primeras tienen que ver con los aspectos 
teóricos y prácticos de la investigación, mientras que en las segundas influyen factores personales, sociales o factores externos a la propia investigación. También existen diversas razones por las que los autores no citan determinados trabajos, como el desconocimiento de su existencia, la imposibilidad de obtenerlos o la inaccesibilidad del idioma en el que los trabajos han sido publicados (Romera, 1996). Tratándose de autores que han colaborado previamente quedan anuladas las razones para no citarse. Asimismo, puesto que se trata de grupos dentro de una misma disciplina científica, llama la atención que manejen corpus científicos no coincidentes, aunque esto se podría matizar ahondando en las líneas de investigación de cada grupo. Por lo que finalmente, las razones para la inexistencia de citas cruzadas entre miembros de diferentes grupos parece responder a factores personales, sociales o factores externos a la propia investigación.

Las citas sirven para medir la influencia de un trabajo sobre los siguientes, pero también la colaboración personal constituye un canal privilegiado de influencia, (Molina, 2002). La novedad del presente trabajo es que se analizan y superponen estos dos tipos de redes, coautoría y citación, sobre los mismos conjuntos de actores, para observar si se establecen los mismos lazos o no, e intentar comprender los agujeros estructurales que se producen.

\section{Material y Métodos}

Las fuentes de información consultadas para el estudio han sido el Social Sciences Citation Index ${ }^{\circledR}$, en adelante SSCl, y el J ournal Citation Reports ${ }^{\circledR}$, Social Sciences Edition, en adelante JCR, productos ambos del Institute for Scientific Information de Philadelphia, hoy Thomson Reuters. Se han elegido estas fuentes por su carácter multidisciplinar dentro de las Ciencias Sociales y su amplia cobertura. Pero sobre todo su elección se justifica en su característica distintiva con respecto a cualquier otra base de datos: sus registros aportan, además de la información bibliográfica considerada básica (autores, lugar de trabajo de los autores, título, fuente, datos de publicación, etc.) las citas que el trabajo en cuestión hace de otros trabajos anteriores. De este modo, cada uno de los registros de la base de datos es, no solo un extracto de información bibliográfica de un trabajo de investigación, sino también una mina de datos relacionales entre investigaciones. Proporciona información de tantas relaciones como citas tiene cada registro. Esta característica la convierte en fuente de información insustituible para un estudio que pretende evidenciar las relaciones entre grupos de investigación. 
Sin embargo, una clasificación temática de los registros del $\mathrm{SSCl}$ plantea problemas. Debido a que no los codifica bajo ninguna clasificación científica y tampoco utiliza un lenguaje de indización controlado. La descripción temática se reduce al campo de palabras clave y a derivar las categorías temáticas que el JCR asigna a las revistas a sus artículos.

EI JCR, el segundo producto del Thompson Reuters utilizado en nuestro estudio, es un directorio de publicaciones científicas, que proporciona información sobre el título completo y abreviado de la revista, el ISSN, la periodicidad, el idioma, y los datos de publicación: editor, dirección y nacionalidad. También, y este es su principal interés para nuestro estudio, clasifica las revistas según una lista de materias que él mismo elabora, explica y actualiza. Además permite a sus usuarios buscar, ordenar y comparar las revistas reseñadas por indicadores bibliométricos reconocidos internacionalmente como el número total de trabajos publicados, las citas que reciben esos trabajos, los índices de Impacto, e Inmediatez, y la Vida Media.

Dado que el JCR es elaborado a partir de una selección de las revistas vaciadas en el $\mathrm{SSCl}$, su interés para nuestro trabajo reside en que clasifica las revistas en un número limitado de materias, subject categories. Se asume pues, que los trabajos contenidos en una revista se pueden clasificar bajo la misma materia o materias que describen a la fuente. El trabajo se ha realizado sobre la producción científica española en Ciencias Sociales, es decir, registros que contuvieran en su campo Address la cadena de texto Spain durante el periodo 1992-2001. Se recuperaron un total de 9229 registros que suponía el volumen total de la producción científica española en el área de Ciencia Sociales. La información para los análisis de citas se extraen del campo Cited references de los registros del $\mathrm{SSCl}$.

Se podría compartimentar la base de datos atendiendo a las materias con las que se clasifican las revistas puesto que se trata de un número limitado y manejable. Sin embargo es posible intuir entre algunas de ellas una proximidad temática mayor que hacia el resto, es decir, subyace en el conjunto una clasificación de un nivel superior, más genérico, que agrupa materias y que hemos denominado en este trabajo Áreas.

Refuerza esta idea el hecho de que en el $\mathrm{SSCl}$ una misma revista pueda estar clasificada en varias materias simultáneamente. Esto implica un vínculo entre esas dos materias, una relación, una proximidad temática, un ámbito de conocimiento interdisciplinar. 
La metáfora de la red, ampliamente utilizada entre los sociólogos, nos permite explicar de forma más sensible, más fiel a la realidad, el establecimiento de las áreas temáticas, que se extienden enredando distintos cuerpos teóricos, metodologías y disciplinas.

El software utilizando para el tratamiento de las matrices y la obtención de gráficos son respectivamente UCINET y Netdraw. Hemos transformado la tabla que relaciona materias con revistas para convertirla en una matriz simétrica ponderada en la que los actores son las materias y las revistas en las que coinciden suponen sus vínculos, el grafo resultante es la figura 1. El tamaño de los nodos representa el volumen de artículos contenidos en las revistas que se clasifican bajo dicha materia y el grosor del vínculo que une los nodos entre sí el número de revistas que comparten ambas materias.

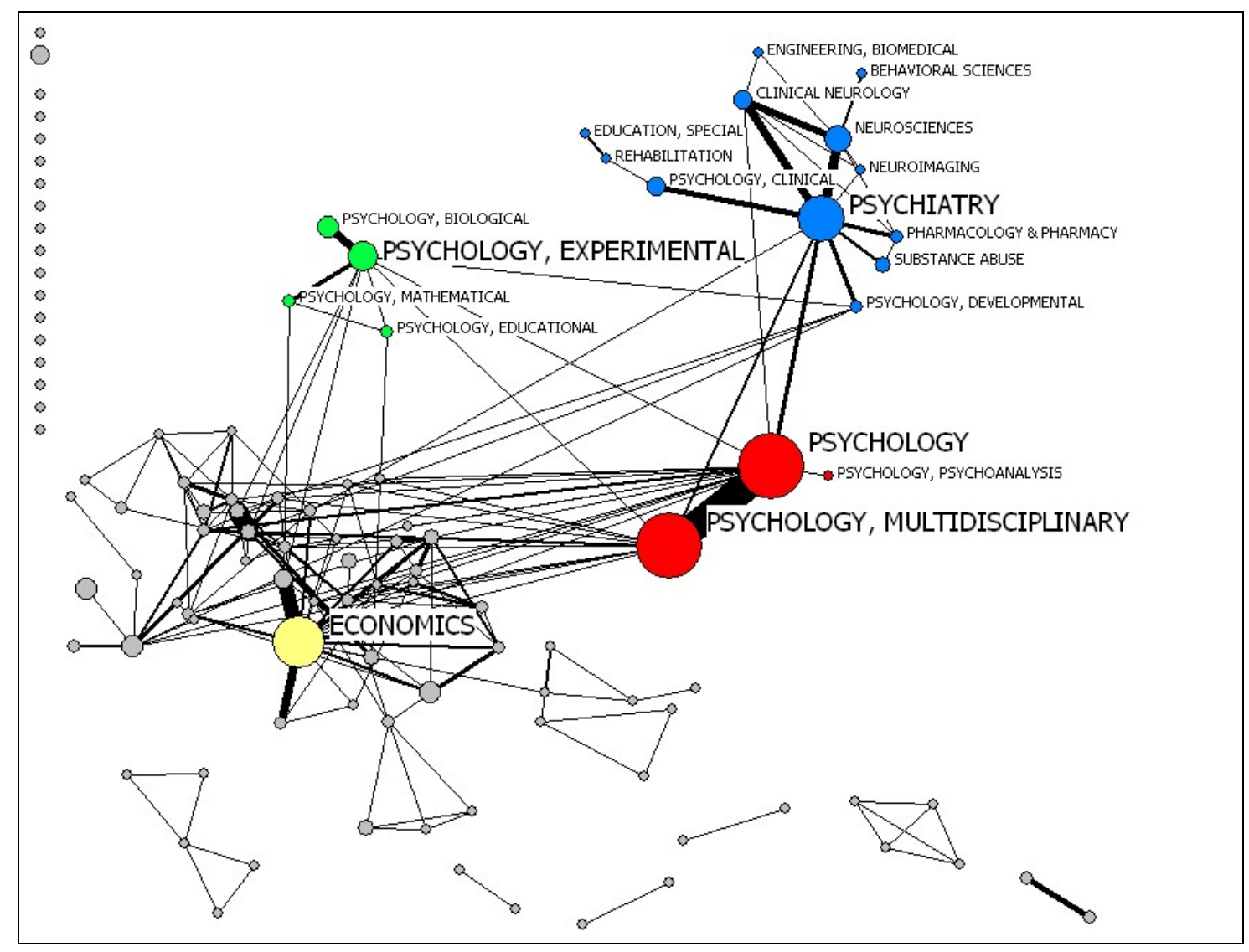

Figura 1. Áreas del SSCI

Este método para seleccionar los trabajos científicos pertenecientes a un área temática escapa a la rigidez de la selección en función de un grupo de materias preconcebido que nunca explica bien los solapamientos entre las mismas y que corre el riesgo de mutilar extensiones de alguna de ellas o simplemente producir silencio ante relaciones incipientes (López, 2009). 
La Tabla 1 resume el peso de las principales áreas sobre el total de la producción científica española en Ciencias Sociales y Humanidades. Para facilitar la comprensión de la figura 1 sólo se muestran las etiquetas de los nodos que representan las categorías temáticas implicadas en nuestra investigación.

\begin{tabular}{lcccc}
\hline \multicolumn{1}{c}{ Área } & Materia & Revista & \multicolumn{2}{c}{ Artículos } \\
\hline Psiquiatría y Neurociencias & 12 & 98 & 1651 & $20 \%$ \\
Psicología General & 3 & 31 & 1555 & $19 \%$ \\
\hline Economía y Empresa & 5 & 93 & 1262 & $14 \%$ \\
Psicología Experimental & 4 & 48 & 725 & $9 \%$ \\
\hline Otras & 68 & 275 & 3156 & $38 \%$ \\
\hline
\end{tabular}

Tabla 1. Indicadores de los entornos de coautoría

A partir de aquí centramos nuestro estudio en las áreas de Psiquiatría, Psicología General y Psicología Experimental por el peso que sobre el total suponen, el 48\%, de la producción científica española en el área de ciencias sociales y humanidades recogida en el SSCl y por su atractivo a la hora de establecer similitudes y diferencias entre ámbitos de investigación tan próximos.

Los estudios bibliométricos aplicados a la Psicología son abundantes. Después de la Medicina, la Psicología es la ciencia que más ha utilizado esta técnica de estudio (Delgado, 2006). Las estrategias de búsqueda aplicadas en estudios bibliométricos precedentes sobre la psicología (Osca, 2005), demuestran que para recoger toda la documentación relacionada con esta ciencia no es posible circunscribirse exclusivamente a lo que bajo el epígrafe de psicología contenga la base de datos en cuestión. Es necesario extender la búsqueda a revistas más propias de otras disciplinas, e incluso a bases de datos, en principio, ajenas a la psicología. Sirva como ejemplo el caso de las bases de datos del Consejo Superior de Investigaciones Científicas, en las que la Psicología se recoge en el ISOC - Ciencias Sociales y Humanidades, la Psiquiatría y la Psicología clínica son recogidas en el IME - Biomedicina, y la Psicofarmacología y la Psicología biológica, obviamente muy relacionadas con la Psiquiatría y la Psicología experimental, son recogidas en la base de datos ICYT - Ciencia y Tecnología.

El conocimiento, más que una yuxtaposición de parcelas, es un espacio continuo en el que se encuentran dispersas las distintas disciplinas científicas, unas más próximas a otras entre sí a través de relaciones que pueden ser representadas en 
una red. Ello supone una novedad en la delimitación de las fronteras de áreas científicas comparables entre sí.

\section{Resultados y Discusión}

Se detallan a continuación los contenidos de las tres áreas temáticas estudiadas. Psiquiatría y Neurociencias agrupa las investigaciones sobre la rama de la medicina que se ocupa de las enfermedades mentales y el sistema nervioso. Se extiende a su metodología de investigación; métodos de diagnóstico, algunos de ellos basados en ingeniería e informática médica; tratamientos farmacológicos y combinación de terapias psicológicas y clínicas; rehabilitación y reeducación de pacientes; especialización sobre los enfermos en distintos grupos de edad; y aspectos sociales de las enfermedades mentales, sobre todo los relacionados con las adicciones.

Psicología General incluye los trabajos de investigación en psicología con un acercamiento general o interdisciplinario al campo. También la psicología filosófica, el psicoanálisis, y la historia de la psicología. Las subject categories que integran esta área no son clases diferentes sino cambios de denominación en las mismas. Esto es algo habitual en esta clasificación de materias, lo que obliga por un lado a realizar continuas actualizaciones en los estudios de actividad científica. Este dinamismo en el sistema de clasificación otorga todavía más valor a una metodología de agrupación temática que tiene en cuenta las relaciones entre las distintas categorías y de esa manera absorbe los cambios que puedan producirse.

Finalmente, Psicología Experimental se centra en los procesos de conciencia, cognición, memoria, y percepción (visual, auditiva, del discurso, etc.) y sus bases biológicas, se incluye la biopsicología, psicofisiología, psicofarmacología, y psicología comparada; también estudia la psicología animal. Y por otro lado, se estudian la metodología e instrumentación de la psicología: métodos multivariables, tratamiento estadístico y estrategias de investigación. Ampliamente relacionados con la estadística, se incluyen, por último, la psicología de la educación y de la escuela, y los test de medición de aprendizaje y comportamiento creativo.

\section{Comparación de las redes de coautoría}

En este estudio se utiliza como variable atributiva de los autores estudiados su producción científica, es decir el número de artículos publicados, ello nos permite justificar la eliminación de algunos actores. Para permitir comparaciones se analiza en todos los casos solamente el grupo de autores que acumulaban aproximadamente el $25 \%$ de toda la producción científica del área. 
En los tres casos se trata de universos completamente distintos en su tamaño y en su configuración. La Psicología Experimental es, en su tamaño, muy diferente de las otras dos áreas analizadas. En ella se han identificado 1139 autores diferentes frente a los 3560 de Psiquiatría o los 2480 de Psicología General. Y el selecto grupo de los más productivos lo forman 58 autores frente a los 146 de Psiquiatría o los 202 de Psicología General.

Por su configuración, encontramos en un extremo la Psiquiatría, en la que el grupo más productivo, aquellos autores que concentran el $25 \%$ del total de la producción científica en el área supone solo el 4,10\% de todos los autores, que están obligados a firmar 9 o más trabajos para pertenecer a él. Además su índice de transitoriedad, el porcentaje de autores ocasionales, es decir, aquellos que firman un solo trabajo, con respecto al total de autores, es notablemente más bajo que en las otras dos áreas temáticas, un $43,54 \%$, lo que es indicativo de materias muy consolidadas. Y en el otro extremo la Psicología General, en la que ese mismo grupo supone el $8,15 \%$ y requiere 4 o más trabajos. Entre uno y otro extremo encontramos la Psicología Experimental en la que sus autores más productivos firman 6 o más trabajos y son el 5,09\% de todos los autores. Sin embargo, las dos psicologías comparten un elevado índice de transitoriedad cercano al $70 \%$.

\begin{tabular}{l|ccc}
\hline & $\begin{array}{c}\text { Psiquiatría y } \\
\text { Neurociencias }\end{array}$ & $\begin{array}{c}\text { Psicología } \\
\text { General }\end{array}$ & $\begin{array}{c}\text { Psicología } \\
\text { Experimental }\end{array}$ \\
\hline & Indicadores bibliométricos & \\
\hline Índice de transitoriedad & $43,54 \%$ & $67,78 \%$ & $66,99 \%$ \\
$\begin{array}{l}\text { Promedio de autores en } \\
\text { cada trabajo }\end{array}$ & 4,1 & 2,7 & 3,1 \\
\hline \multicolumn{4}{c}{ Medidas de centralización de los gráficos de coautoría } \\
\hline Densidad & $15,49 \%$ & $3,57 \%$ & $24,74 \%$ \\
Inclusión & $93,15 \%$ & $79,70 \%$ & $84,48 \%$ \\
\hline
\end{tabular}

Tabla 2. Indicadores de los entornos de coautoría

La tasa de coautoría o firma conjunta en publicaciones científicas es un indicador de colaboración científica. Concuerda con lo explicado anteriormente sobre la configuración de las tres áreas temáticas, el promedio más alto de autores en cada trabajo lo ostenta la Psiquiatría con un 4,1, seguido de la Psicología Experimental, 
con 3,1 firmas por trabajo, y, finalmente, Psicología General tiene un promedio de 2,7 firmas por trabajo.

La densidad e inclusión son cálculos sobre el conjunto del entorno social, incluyen todos los componentes que lo integran y también sus nodos aislados y aportan, por lo tanto, valores de comparación entre gráficos que representen, a su vez, distintos entornos sociales. Ambas medidas, presentadas en la tabla 2 corresponden a los gráficos que representan la colaboración entre los autores del cuartil más productivo de cada disciplina.

La densidad mide la cantidad de vínculos que se dan entre los nodos que integran un mismo entorno; se expresa en forma de porcentaje sobre el total de vínculos posibles. Un gráfico estaría completo si absolutamente todos los nodos que lo integran estuvieran vinculados al resto de nodos, lo que, como es fácil prever, es poco probable, sobre escenarios reales. La inclusión es el porcentaje de nodos que están conectados sobre el total de nodos del gráfico. Aquí la clave son los nodos aislados, cuanto menor sea el número de nodos aislados mayor será el grado de inclusión del gráfico.

En estas figuras volvemos a observar, como primera diferencia, el tamaño del universo de Psicología Experimental, mucho más reducido, frente a Psiquiatría o Psicología General.

La comparación de las medidas de centralización entre Psiquiatría y Neurociencias, Psicología General, y Psicología Experimental señala notables diferencias en la forma de colaboración que establecen sus investigadores. Los datos densidad e inclusión muestran porcentajes altos para Psiquiatría y bajos para Psicología General lo que está en correspondencia con las tasas de coautoría: 1,4 puntos de diferencia de la primera sobre la segunda. 


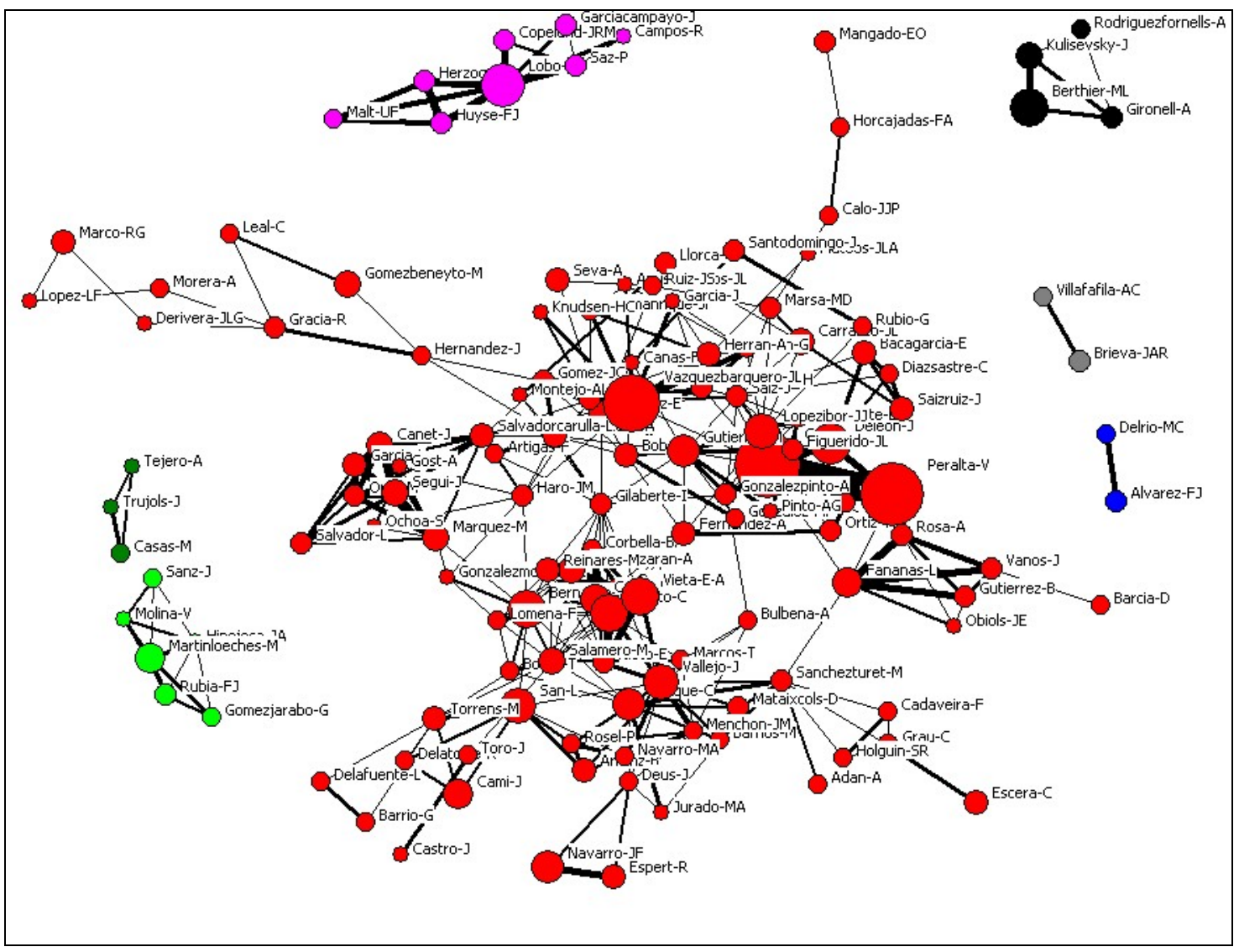

Figura 2. Coautoría en Psiquiatría y Neurociencias

La figura 2 muestra un área temática extensa con gran cantidad de autores y múltiples relaciones entre ellos. De las distintas agrupaciones de nodos posibles, la más simple es el componente, el mayor subconjunto posible de nodos y sus vínculos en el que todos los nodos que lo integran estén conectados al menos a otro nodo del subconjunto y en el que es posible llegar desde y hasta cualquier nodo del componente siguiendo los vínculos que contiene. En la figura se puede observar como en el entorno social de la colaboración de los investigadores españoles más productivos en Psiquiatría y Neurociencias casi todos ellos están integrados en un mismo componente, por ello presenta la densidad más alta de las tres áreas temáticas estudiadas, un 15,49\%. Al margen de este componente principal se distinguen solo otras dos estructuras que podamos considerar clusters, nodos entre los que se da una alta densidad en las relaciones (Herrero, 2000) por la multilateralidad de sus relaciones.

La inclusión es más alta para Psiquiatría y más baja para Psicología General. Esta medida indica que aproximadamente un $20 \%$ de los investigadores principales y más productivos de Psicología General permanecen totalmente aislados del resto de 
investigadores durante todo el extenso periodo estudiado. Esta misma medida es, sin embargo, de apenas un 7\% en Psiquiatría.



Figura 3. Coautoría en Psicología General

La figura 3, que representa la colaboración en Psicología General, presenta, en contraposición a la Psiquiatría y Neurociencias, muchos componentes, numerosos grupos de trabajo o colaboración pequeños, bien cohesionados entre sí pero totalmente aislados unos de otros, por eso la densidad del gráfico es muy baja, de un $3,57 \%$.

En la mayoría de los casos, los autores más productivos pertenecen a distintos componentes entre los que no hay colaboración lo que indicaría un elevado nivel de competitividad entre ellos. $Y$ en el caso en el que se dan varios máximos productores en un mismo componente se observa que estos autores establecen vínculos más fuertes con otros autores de menor producción científica que ellos, lo que indica que mantienen subgrupos de trabajo más o menos independientes. 


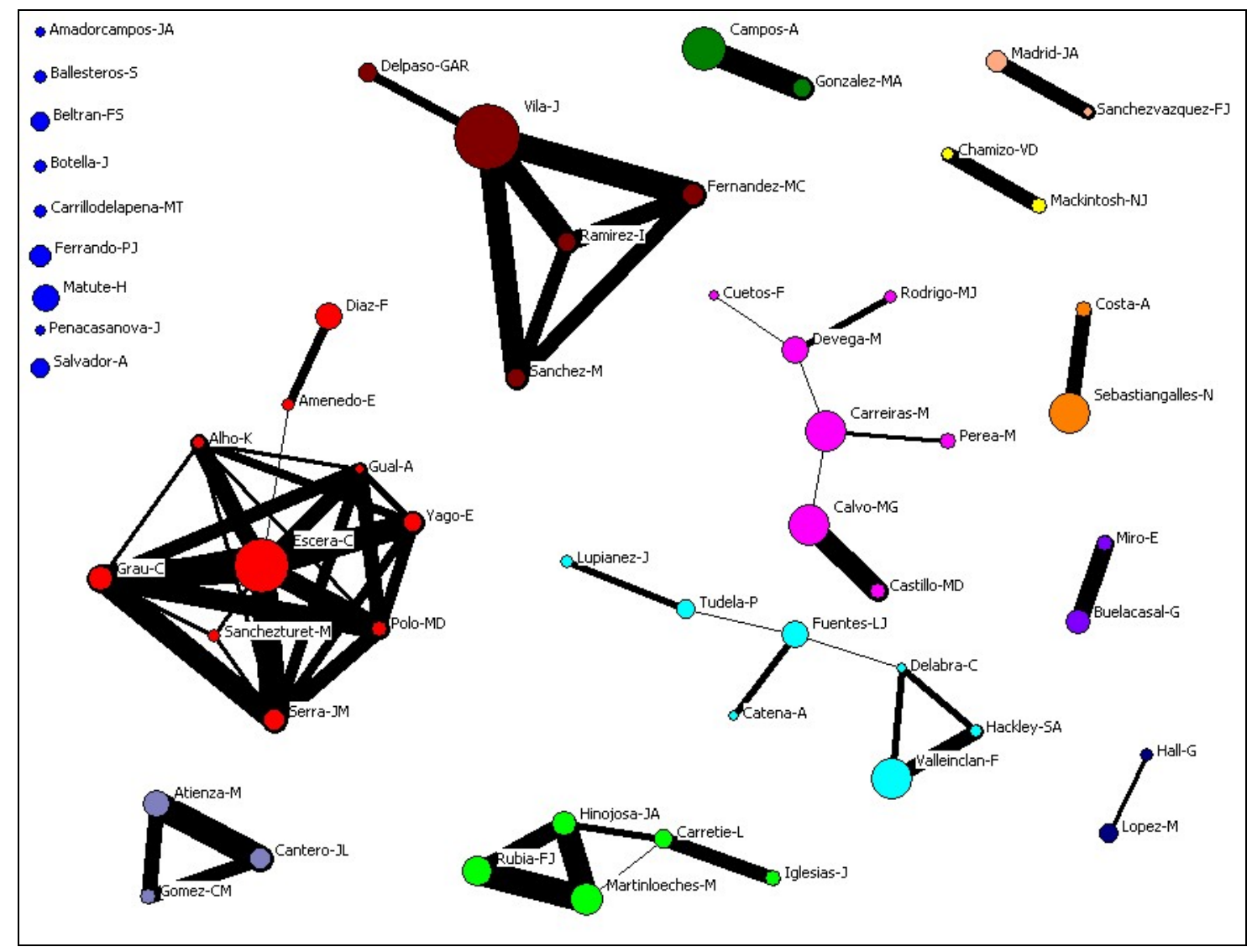

Figura 4. Coautoría en Psicología Experimental

Una vez más encontramos entre dos aguas la Psicología Experimental, presenta valores intermedios entre las otras dos áreas temáticas estudiadas en el promedio de autores que firman cada trabajo, 3,1, y el índice de transitoriedad, un 66,99\%. Tal y como ocurría con la Psicología General, presenta muchos componentes totalmente diferenciados entre sí, pero con una densidad del gráfico mucho más alta, del 24,74\%. La tasa de coautoría, más alta que en Psicología General, da lugar a componentes de mayor tamaño, además el universo de autores disponibles con los que colaborar es más limitado, y las relaciones establecidas se repiten con más frecuencia. Ambas cuestiones repercuten positivamente en una mayor densidad del gráfico.

Con respecto a la inclusión, un $15 \%$ de los autores de Psicología Experimental permanecen desconectados de su entorno, un porcentaje menor que en Psicología General, que alcanza hasta el $20 \%$.

\section{Comparación de las redes de citas}

Si tenemos en cuenta que el promedio de citas que incluye cada registro está por encima de 30 , se entiende que cualquier volumen de referencias trabajado se 
convierte rápidamente en inabarcable cuando pasamos al análisis de citas. Además no es posible llevar a cabo ningún estudio concluyente sobre las citas tal y como se obtienen de la base de datos. En cada cita de cada registro de la base de datos se acumulan en una única cadena de texto informaciones que es preciso tratar por separado. Dicha cadena de texto agrupa, en un solo cuerpo el nombre del autor, el año de publicación, la revista en la que se publicó el trabajo que se está citando, el volumen, y la página de comienzo del artículo. El objeto de esta cita es identificar de forma tan breve como sea posible un trabajo científico y aunque en principio el resultado parece bueno, dada la ausencia de control en las formas de los nombres de autor y las abreviaturas de las revistas, es posible rastrear en la base de datos el mismo trabajo citado con muchas variantes.

Los trabajos sobre pautas de citación requieren que los autores puedan ser tratados unitariamente y no dispersos en las citas que reciben a sus diferentes trabajos. Por todo ello en esta investigación se ha llevado a cabo un trabajo de normalización previo a la elaboración de cálculos y figuras en el que se han agrupado todas las citas que recibe un mismo autor, independientemente de a qué artículo en concreto se esté citando.

Como ya se anticipó en el apartado de material y métodos, lo más interesante de un ARS aplicado a las citas es que permitiría evidenciar las relaciones entre grupos de investigación. Las tres áreas, se apoyan en publicaciones extranjeras, fundamentalmente europeas y americanas. Por ejemplo, en psicología aproximadamente la mitad de las monografías publicadas son traducciones de obras extranjeras (Prieto, 1994). Sin embargo, ante la cantidad de datos relacionales que el conjunto de todas las citas proporciona y, de acuerdo con los objetivos de la presente investigación, la matriz para el análisis reticular se ha construido con los autores más productivos de cada área temática estudiada y sus citas entre ellos.

El número medio de referencias que los artículos aportan en sus bibliografías es diferente, véase la tabla 3. La Psiquiatría es la materia que más citas suele llevar, un promedio de 34 citas por trabajo, con notable diferencia sobre las psicologías. De nuevo la Psicología Experimental con 25 referencias se encuentra a mitad de camino entre la Psiquiatría y la Psicología General que aporta un promedio de 21 referencias. 


\begin{tabular}{lccc}
\hline & $\begin{array}{c}\text { Psiquiatría y } \\
\text { Neurociencias }\end{array}$ & $\begin{array}{c}\text { Psicología } \\
\text { General }\end{array}$ & $\begin{array}{c}\text { Psicología } \\
\text { Experimental }\end{array}$ \\
\hline $\begin{array}{l}\text { Indicadores bibliométricos } \\
\text { bromedio de referencias }\end{array}$ & 34 & 21 & 25 \\
\hline \multicolumn{4}{c}{ Medidas de centralización de los gráficos de redes de citas } \\
\hline Densidad & $32,78 \%$ & $8,69 \%$ & $12,24 \%$ \\
Inclusión & $85,71 \%$ & $56,25 \%$ & $57,14 \%$ \\
\hline
\end{tabular}

Tabla 3. Indicadores de las redes citas

La comparación de las medidas de centralización de los gráficos que representan las redes de citas en las tres áreas estudiadas demuestra que las psicologías comparten un patrón de citación muy parecido y notablemente diferente al de la Psiquiatría. Esto confirma lo que intuitivamente se observaba en los gráficos: en las psicologías las pautas de citación alinean las colaboraciones en muchos componentes desconectados entre sí sobre los que destaca la cohesión de uno con mucha diferencia sobre el resto, mientras que en Psiquiatría prácticamente todos los miembros están conectados en un mismo componente, aunque la frecuencia y flujo de las uniones permita distinguir claramente las dos facciones diferenciadas que secundan las pautas de citación.

En las figuras 5, 6 y 7, el tamaño de los nodos indica el número total de citas que un autor recibe, incluyendo las autocitas. Los vínculos y la dirección de sus flechas indican de qué autores provienen las citas que reciben y el grosor de las líneas su cantidad, de tal manera que el prestigio de un autor no se mide por el tamaño absoluto del nodo que lo representa, sino por la cantidad y grosor de líneas que llegan a él apuntándole con una flecha. 


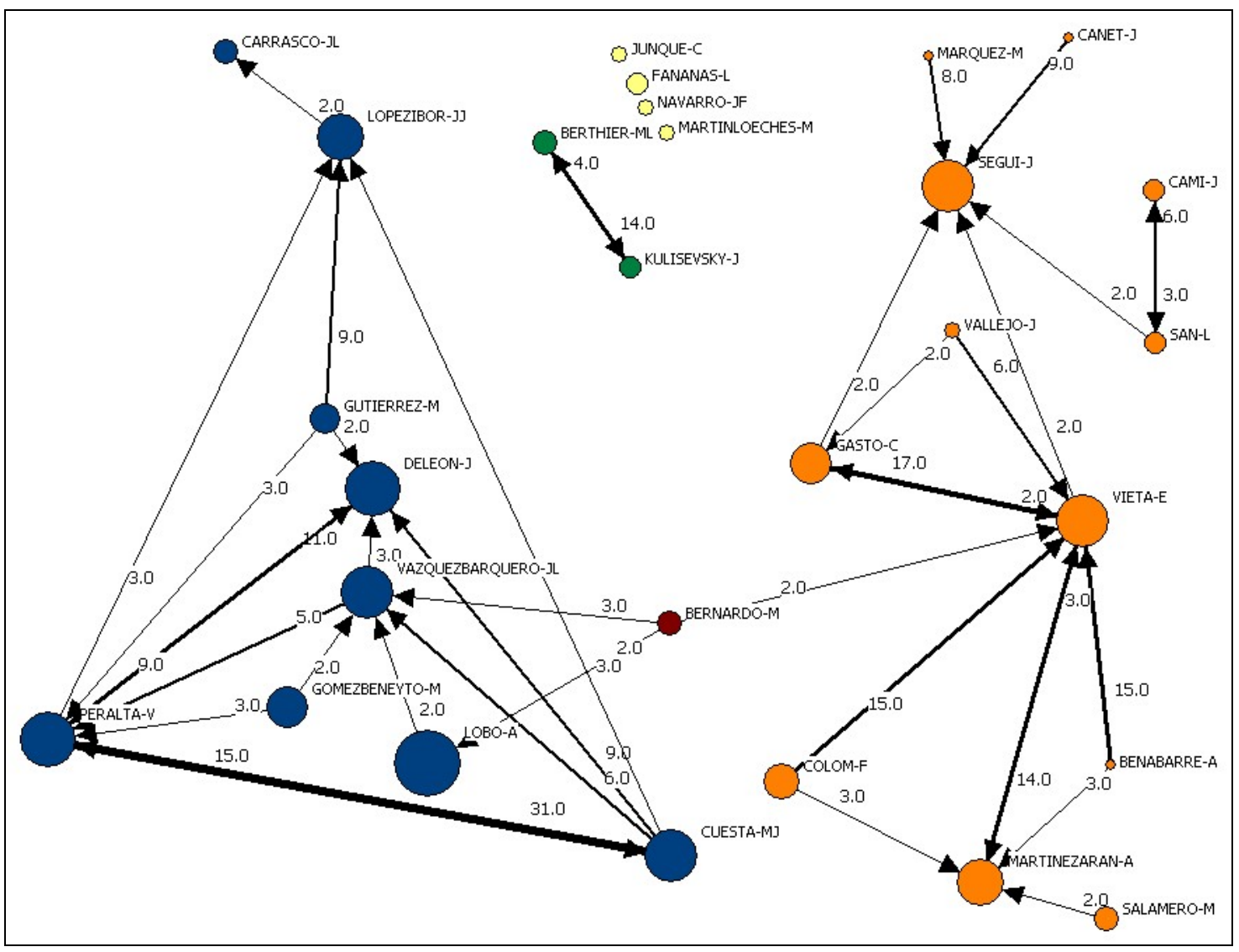

Figura 5. Redes de citas en Psiquiatría y Neurociencias

En el área de Psiquiatría y Neurociencias, de todas las relaciones posibles entre los nodos que aparecen en el gráfico se establecen un 32,78\%, esta es una medida de densidad muy alta en comparación con las de las psicologías. Y hasta el $85,71 \%$ de los nodos establece relación con otros miembros del área, es decir, una tasa de inclusión muy alta.

En este área, ver figura 5, se distinguen dos subgrupos totalmente independientes que marcan las pautas de citación en la disciplina. Los autores que integran cada subgrupo circunscriben sus citas a otros miembros de su propio grupo, manteniéndose separados del otro subgrupo. No podemos hablar, no obstante, de componentes independientes porque existe un único autor que establece conexiones con miembros de ambos subgrupos, al que, por cierto, no cita ningún autor de dichos subgrupos.

El vínculo más grueso de todo el gráfico, que indica una relación basada en las citas mutuas se establece entre dos autores que si volvemos al gráfico de colaboración observamos que son los dos máximos productores del área, miembros del mismo grupo de investigación, y además, autores estructuralmente equivalentes. 
Al margen de estos dos subgrupos existen otro grupo cuyos miembros se citan entre sí al margen del resto y que, también superponiendo esta red con la de colaboración nos permite observar que en todos los casos son miembros de un grupo de trabajo.

Tanto en Psicología General como en Psicología Experimental las medidas de centralización son parecidas, respectivamente un $56,25 \%$ y un $57,14 \%$ de inclusión, lo que supone que más de un $40 \%$ de los investigadores permanece fuera del entorno de citación. Y tan solo un 8,69\% para la Psicología General y un 12,24\% para la Psicología Experimental de densidad.



Figura 6. Redes de citas en Psicología General

Como ya se ha avanzado anteriormente en este trabajo, en Psicología General las pautas de citación muestran un escenario con muchos componentes desconectados entre sí sobre los que destaca la cohesión de uno con mucha diferencia sobre el resto.

En todos los casos se comprueba que los nodos que integran un componente en el gráfico de citas coinciden a su vez en un mismo componente del gráfico de colaboración, esto quiere decir que los investigadores citan a otros miembros de 
sus grupos de trabajo y a su vez permanecen aislados del resto, es decir, que no citan a investigadores de otros grupos.

Al margen del componente principal de la figura 6, el resto de componentes son pares de nodos de los que solo uno de ellos recibe citas, estos receptores son, si volvemos al gráfico de grupos de trabajo, líderes de sus respectivos equipos.

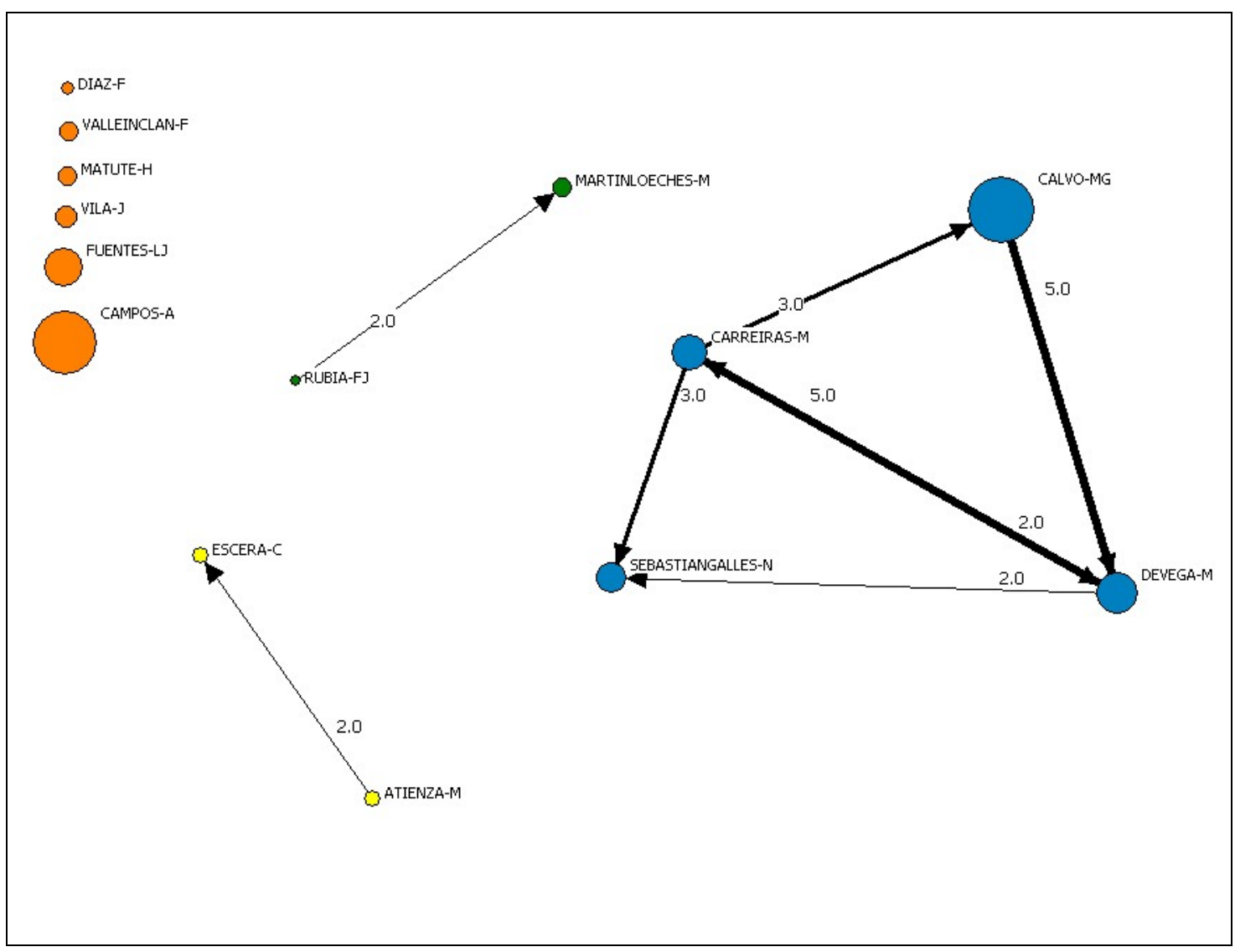

Figura 7. Redes de citas en Psicología Experimental

Finalmente, los flujos de citación en la Psicología Experimental son muy similares a los de la Psicología General, como ya se podía prever por sus medidas de centralización. El componente principal está integrado por autores que son además miembros de un mismo componente en la figura 4. En los otros componentes se distinguen los roles de líder en cuanto que autor citado dentro del componente.

Sin embargo es posible aportar una diferencia con respecto a los patrones vistos hasta ahora y es el caso de un nodo del componente principal que recibe citas de otros miembros del componente y, sin embargo, al superponer la red de coautoría observamos que forma parte de otro equipo de trabajo. 


\section{Conclusiones}

Las conclusiones de la presente investigación, de acuerdo con los objetivos que se propusieron, son:

1. El ARS aplicado a los sistemas de clasificación de las bases de datos es útil para acotar universos de conocimiento que trascienden los propios sistemas de clasificación siempre más simplistas. La unidimensionalidad de las listas de materias, bajo las que cada base de datos clasifica las revistas que vacía o directamente los registros que contiene, no son suficientemente flexibles como para representar las relaciones que se establecen entre las distintas ciencias.

Así este trabajo ha demostrado las relaciones que, por compartición de títulos de revista, se establecen entre las materias [subject categories] Behavioral Sciences; Clinical Neurology; Special Education; Biomedical Engineering; Neuroimaging; Neurosciences; Pharmacology \& Pharmacy; Psychiatry; Clinical Psychology; Developmental Psychology; Rehabilitation; y Substance Abuse configuran el área temática de Psiquiatría y Neurociencias. Por la misma razón las materias Psychology; y Multidisciplinary Psychology, configuraron el área Psicología General y multidisciplinar. Y, finalmente, las materias Biological Psychology; Educational Psychology; Experimental Psychology; y Mathematical Psychology, configuran el area Psicología Experimental.

2. La utilización complementaria de las técnicas bibliométricas y el ARS se ha desvelado en la presente investigación como mucho más útil que lo que se podría esperar de la simple suma de dos métodos distintos.

Los gráficos de redes permiten objetivar grupos de trabajo que de otro modo sería difícil descubrir. Para la delimitación de lo que es un equipo es tan importante la inclusión como la exclusión de miembros a través de relaciones, lo que en ARS se conoce como agujeros estructurales. $Y$ esto se ha podido comprobar por la superposición de las redes de colaboración con las de citas. Las primeras representan relaciones explícitas y las segundas relaciones implícitas, pero ambas sirven para posicionar autores y grupos entre sí.

Se ha utilizado el indicador más consagrado para la evaluación de la actividad investigadora, la producción científica, para justificar la eliminación de algunos actores, los de más baja producción, para permitir gráficos manejables. Y también como atributo principal de los nodos en el ARS colaboración lo que ha sido de gran ayuda para identificar posiciones equivalentes, es decir, autores 
que ostentan el mismo rol dentro de cada grupo de trabajo, por ejemplo los líderes de cada equipo.

3. Se constata lo que hace ya tiempo se reclama, especialmente por los investigadores de las ciencias sociales y las humanidades: la necesidad de una flexibilización en los baremos de evaluación de las diferentes áreas científicas. Dicha flexibilidad debería tener en cuenta los matices y características propias de las diversas disciplinas que se integran institucional y conceptualmente bajo macrocategorías científicas que eluden la superespecialización e interdisciplinariedad actual y para cuyo análisis se ha descubierto como gran herramienta el ARS.

En las áreas científicas analizadas en este trabajo se ha probado que se trata de universos completamente distintos en su tamaño y en su configuración. La Psicología Experimental es, en su dimensión, muy diferente de las otras dos áreas analizadas. En cuanto a la configuración de sus redes, tanto las de colaboración como las de citas, encontramos dos modelos diferentes uno para la Psiquiatría y otro que comparten las dos psicologías.

En Psiquiatría, prácticamente todos los investigadores están incluidos en algún grupo de trabajo, y la mayoría de ellos están conectados en una misma estructura a través de su colaboración en los trabajos realizados. También las pautas de citación muestran un entorno en el que la mayoría de sus integrantes se reconocen mutuamente sus aportaciones a pesar de que sea posible distinguir por la frecuencia y flujo de las uniones facciones diferenciadas en los grupos de influencia.

En el lado opuesto encontramos la Psicología General en la que hasta una quinta parte de los investigadores principales y más productivos permanecen aislados, fuera de las redes de colaboración, y el entorno presenta, en cuanto a su configuración, multitud de componentes, es decir, numerosos grupos de trabajo bien cohesionados entre sí pero totalmente aislados unos de otros. Esta desconexión entre los distintos grupos es todavía más acusada en el comportamiento mostrado en los hábitos de citación, no hay reconocimiento fuera de los miembros del propio equipo de trabajo y además los roles de unos investigadores con respecto a otros es mucho más acusado: la dirección de las citas refuerza el papel de solo uno de los nodos del componente, nodo que coincide en los gráficos de colaboración con el autor más productivo y que hemos identificado como líder del grupo. 
Finalmente se apuntan las futuras líneas de investigación que se han planteado a la finalización del presente trabajo.

Una aplicación seria del ARS en los estudios sociométricos de la actividad científica, que no se conformase sólo con la innovación que supone aportar nuevas formas de representación de los datos, podría dar como resultado nuevos sistemas de evaluación, mejores, más complejos y que se adaptaran con más acierto a las particularidades de distintas áreas temáticas, zonas geográficas y culturas científicas. Para ello sería necesario llevar a cabo estudios descriptivos y comparativos de envergadura que permitieran obtener conclusiones fiables, de los que el presente trabajo sólo puede considerarse un ensayo. Algunas de las preguntas a responder podrían ser si la estructura en los grupos de investigación condiciona sus resultados, y si podría destacarse una configuración concreta como idónea.

También se plantean dudas sobre los contenidos de las relaciones que utilizamos actualmente, coautoría y citación, y sobre las fuentes empleadas. Dicho de otro modo ¿la firma conjunta de trabajos supone realmente colaboración científica y las citas una utilización previa de conocimientos? Y aún en caso afirmativo ¿en todos los casos? Quizás sería oportuno contrastar esos datos con encuestas a los investigadores. A pesar de que las fuentes de información que se utilizan en los estudios de actividad científica son preexistentes y creadas para otros fines hace mucho que los investigadores adaptan sus comportamientos para obtener mejores resultados en el sistema de reconocimiento de méritos, especialmente en las áreas científicas que siempre se han sentido desfavorecidas en una comparación entre disciplinas. Para corregir esto habría que enriquecer y diversificar el origen de datos, y vincular los estudios bibliométricos, es decir, de rendimiento de actividad científica a los de input de recursos.

\section{Bibliografía}

Acedo, FJ, Barroso, C, Casanueva, C, Galan, JL (2006). Co-authorship in management and organizational studies: An empirical and network analysis. J ournal of Management Studies, vol. 43, no 5, pp. 957-983.

Boyack, KW, Klavans, R, Borner, K (2005). Mapping the backbone of science. Scientometrics, vol. 64, no 3, pp. 351-374.

Bozeman, B, Corley, E (2004). Scientists' collaboration strategies: Implications for scientific and technical human capital. Research Policy, vol. 33, № 4, pp. 599-616. 
Casanueva Roche, C, Escobar Pérez, B, Larrinaga González, C. (2007). Red social de contabilidad en españa a partir de los tribunales de tesis. Revista Española de Financiación y Contabilidad, vol. XXXVI, no 136, pp. 707-726.

Delgado López-Cózar, E, Torres-Salinas, D, Jiménez-Contreras, E, Ruiz-Pérez, R. (2006). Análisis bibliométrico y de redes sociales aplicado a las tesis bibliométricas defendidas en españa (1976-2002): Temas, escuelas científicas y redes académicas. Revista Española de Documentación Científica, vol. 29, no 4, pp. 493424.

Freeman, L.C (2000). La centralidad en las redes sociales. Clarificación conceptual. Política y Sociedad, no. 33, pp. 131-148.

Herrero, R (2000). La terminología del análisis de redes. Problemas de definición y de traducción. Política y Sociedad, no 33, pp. 199-206.

Iñiguez, L, Muñoz Justicia, J, Peñaranda, MC, Martínez, LM (2006). La psicología social en España: estructuras de comunidades. Redes: Revista Hispana para el Análisis de Redes Sociales, vol. 10, № 3 .

Jiménez Contreras, E. (2000). Los métodos bibliométricos: estado de la cuestión y aplicaciones. En: Cuadernos de Documentación Multimedia, no 10, pp. 1575-9733

Jiménez, F, Zabala-Iturriagagoitia, JM, Zofío, JL (2007). Efficiency in public research centers: Evaluating the Spanish food technology program. Working Papers in Economic Theory. [http://ideas.repec.org/p/uam/wpaper/200704.html]

Katz, JS,, Martin, BR (1997). What is research collaboration? Research Policy, vol. 26, no 1 , pp. 1-18.

Leydesdorff, $L$ (2004). Clusters and maps of science journals based on bi-connected graphs in journal citation reports. Journal of Documentation, vol. 60, no 4, pp. 371427.

Leydesdorff, L, Rafols, I (2009). A global map of science based on the ISI subject categories. Journal of the American Society for Information Science and Technology, vol. 60, no 2, pp. 348-362.

López Ferrer, MT (2009). Aplicación del análisis de redes a un estudio bibliométrico sobre psiquiatría, psicología general y psicología experimental. València: Universitat de València. ISBN 978-84-370-7245-6.

Lotka, AJ (1926). The frequency distribution of scientific productivity. J ournal of the Washington Academy of Science, vol. 16, pp. 317-323.

Molina, JL (2001). El análisis de redes sociales. Una introducción. Barcelona: Edicions Bellaterra.

Molina, JL (2009). Panorama de la investigación en redes sociales. REDES - Revista hispana para el análisis de redes sociales, vol. 17, no 11, pp. 235-256.

Molina, JL, Muñoz Justicia, J, Domènech Argemí, M (2002). Redes de publicaciones científicas. Un análisis de la estructura de coautorías. Redes: Revista hispana para el análisis de redes sociales, vol. 1, no 3 . 
Moya-Anegón, F de, Vargas-Quesada, B, Herrero-Solana, V, Chinchilla-Rodríguez, Z, Corera-Álvarez, E, Muñoz-Fernández, FJ (2004). A new technique for building maps of large scientific domains based on the cocitation of classes and categories. Scientometrics, vol. 61, no 1, pp. 129-145.

Olmeda-Gómez, C (dir.), et al. (2006). La investigación en colaboración de las universidades españolas (2000)- (2004). Madrid: Ministerio de Educación y Ciencia.

Olmeda-Gomez, C, Perianes-Rodriguez, A, Ovalle-Perandones, MA, Guerrero-Bote, VP, de Moya-Anegón, F de. (2009). Visualization of scientific co-authorship in Spanish universities: from regionalization to internationalization. Aslib Proceedings, vol. 61, no 1 , pp. 83-100.

Olmeda-Gómez, C, Perianes-Rodríguez, A, Ovalle-Perandones, MA, Moya-Anegón, F de. (2009). Colegios visibles: Estructuras de coparticipación en tribunales de tesis doctorales de biblioteconomía y documentación en españa. El Profesional de la Informacion, vol. 18, no 1, pp. 41-49.

Osca Lluch, J, Civera, C, Tortosa Gil, F, Quiñones, E, Peñaranda, M, López, JJ (2005). Difusión de las revistas españolas de psicología en bases de datos nacionales e internacionales. Anales de Documentación, no 8, pp. 165-186.

Perianes-Rodríguez, A, Olmeda-Gómez, C, Moya-Anegón, F de (2010). Detecting, identifying and visualizing research groups in co-authorship networks. Scientometrics, vol. 82, no 2, pp. 307-319.

Prieto, JM, Fernández Ballesteros, R, Carpintero Capell, H (1994) Contemporary psychology in Spain. Annual Review of Psychology, vol. 45, pp. 51-78.

Pritchard, A (1969). Statistical bibliography or bibliometrics? Journal of Documentation. vol. 25, no 4, p. 348-349.

Requena Santos, F (2003). Análisis de redes sociales: orígenes, teorías y aplicaciones. Madrid: Centro de Investigaciones Sociológicas.

Rodríguez, JA (2005). Análisis estructural y de redes. 2a ed. Madrid: Centro de Investigaciones Sociológicas.

Romera Iruela, MJ (1996). Citas y referencias bibliográficas en el sistema de comunicación científica. Revista Complutense de Educación, vol. 7, no 1, pp. 243270.

Sierra, G (2003). Deconstrucción de los tribunales del CSIC en el período 19852002: Profesores de investigación en el área de física. Apuntes de Ciencia y Tecnología, no 7, pp. 30-38.

Vinkler, P (1987). A quasi-quantitative citation model. Scientometrics, vol. 12, no 1 , pp. 47-72.

Wasserman, S, Faust, K (1999). Social Networks Analysis: Methods and Applications. New York: Cambridge University Press. 